\title{
Racial Parity in Pancreatic Cancer Treatment and Outcomes within an Integrated Healthcare System
}

\author{
Diana S Hsu ${ }^{1 *}$, Sidney $T$ Le $^{1}$, Alex Chang ${ }^{2}$, Austin L Spitzer ${ }^{2}$, George Kazantsev ${ }^{2}$, Peter D \\ Peng ${ }^{2}$ and Ching-Kuo Chang ${ }^{2}$ \\ ${ }^{1}$ Department of Surgery at Highland Hospital, University of California, San Francisco-East Bay, USA \\ ${ }^{2}$ Department of Surgery, Kaiser Permanente Oakland Medical Center, USA
}

\begin{abstract}
Background: This study evaluates survival outcomes of pancreatic ductal adenocarcinoma patients treated at a large, integrated health system and whether there are differences based on race.

Method: We conducted a retrospective study from 2010-2018 among patients at Kaiser Permanente Northern California (KPNC). Self-reported racial ethnic groups included Caucasian, African American, Hispanic, Asian, and Other. Kaplan Meier and Cox regression analyses were used to evaluate differences in race, age, stage, and treatment utilization in relation to overall survival.
\end{abstract}

\begin{abstract}
Results: A total of 3,799 patients were diagnosed with pancreatic ductal adenocarcinoma from 2010-2018. The mean age of Caucasians was on average 2.5 years higher than the mean age of non-Caucasians $(p<0.001)$. The difference in mean overall survival (OS) between the race groups was not statistically significant. Kaplan-Meier survival analyses was performed and demonstrated statistically significant differences in mean OS between age groups $(p<0.001)$. OS was highest, 44.0 months, for those who underwent surgery, chemotherapy, and radiation.
\end{abstract}

Conclusion: This study identified racial parity in survival outcomes and treatment utilization for pancreatic cancer patients who were treated at a large, integrated health care system where patients have equal access to care.

\section{Keywords}

Racial parity, Outcomes, Pancreatic cancer, Integrated healthcare system

\section{Introduction}

Pancreatic cancer is the $12^{\text {th }}$ most common cancer in the world, accounting for $2.7 \%$ of all cancers diagnosed [1], and it is the $3^{\text {rd }}$ leading cause of death by cancer in the United States. 1-year and 5-year survival rates, combining all stages, is $27 \%$ and $6 \%$, respectively [1]. Not only does pancreatic cancer have an overall low survival rate, multiple studies have shown that African Americans diagnosed with pancreatic cancer have worse outcomes. African American patients are less likely to be recommended surgery, to undergo surgery when recommended (OR 0.66), and to receive adjuvant therapy [2-4]. When they undergo surgery, they have a higher mortality compared to Caucasian patients (OR1.27, 95\% Cl: 1.01-1.61) [5,6]. Being of non-Hispanic black race has been identified as an independent predictor for pancreatic cancerrelated death [7]. Some have shown that worse outcomes for African American patients may be in part due to race being closely correlated to or serving as a placeholder for low socioeconomic status [6-10]. Studies have also identified differences in distribution of tobacco use and obesity in patients of different races diagnosed with pancreatic cancer, speculating that increased pancreatic cancer risk and worse pancreatic-cancer related survival seen in African American patients could be partly attributed to differences in habits and habitus $[11,12]$.

However, it is uncertain whether these disparities also stem from treatment inequalities, such as time from diagnosis to surgery, percentage of patients undergoing neoadjuvant chemotherapy, and likelihood of receiving surgery, in addition to a combination of the aforementioned factors.

*Corresponding author: Diana S Hsu, Department of Surgery at Highland Hospital, University of California, San Francisco-East Bay, 1411 East $31^{\text {st }}$ Street, QIC 22134, Oakland, CA 94602, USA; Tel: (+1) 510-437-4078

Accepted: August 27, 2021

Published online: August 29, 2021

Citation: Hsu DS, Le ST, Chang A, et al. (2021) Racial Parity in Pancreatic Cancer Treatment and Outcomes within an Integrated Healthcare System. J Pancreat Cancer Treat 3(1):14-21 
We therefore seek to investigate racial disparity in pancreatic cancer treatment and outcomes in a large integrated health care system, Kaiser Permanente Northern California (KPNC).

\section{Materials and Methods}

\section{Data source and patient selection}

A retrospective cohort study was conducted using data from the KPNC cancer registry data system. KPNC serves a total population of approximately 3.9 million Kaiser Foundational Health Plan, Incorporated members who are cared for by the 8,500 physicians of The Permanente Medical Group, Inc. across 21 hospitals, under a mutual exclusivity arrangement. All KPNC hospitals and clinics employ the same information systems and use Epic electronic medical record (EMR) system. The KPNC cancer registry is a northern California database maintained by the integrated 21-hospital system and Kaiser Health Plan, and reports information on all reportable cancers to the State of California and the Surveillance, Epidemiology, and End Results (SEER) program. The population studied includes all patients under the Kaiser heath plan with pancreatic cancer seen at any Kaiser health facility in Northern California. Follow-up is reported to the registry with mandatory data points including date of last contact, vital status, and cancer status.

A total of 4,340 pancreatic cancer patients were identified between February 2010 and December 2018. Of those, 3,799 were diagnosed with pancreatic adenocarcinoma. Benign disease, premalignant cystic disease, neuroendocrine tumors, lymphomas and metastatic lesions were excluded.

\section{Variable definitions}

The main independent variable (categorical) was the self-reported race of the patient. Race was divided into five subgroups: African-American, Hispanic, Caucasian, Asian (Chinese, Japanese, Filipino, Hawaiian, Korean, Vietnamese, Laotian, Hmong, Kampuchean, Thai, Asian Indian, Pakistani, Micronesian, Chamorro, Guamanian, Polynesian, Tahitian, Samoan, Tongan, Melanesian, Fiji Islanders, New Guinean, Other Asian, and Pacific Islander NOS), and Other. The outcome was a numerical variable defined as "time to death". This was based on the difference in months from the date of diagnosis reported and the date of death, which was confirmed by death certificates on the National Death Index.

Potential confounders considered included age in years at diagnosis (<50, 50-59, 60-69, 70-79 and > 80), sex (male or female), stage at diagnosis (in situ, localized, regional, or distant), tumor location (body, head, tail, NOS), treatment received (surgery, chemotherapy, radiation, surgery and chemotherapy, surgery and radiation, chemotherapy and radiation, all, or none).

\section{Statistical analysis}

Descriptive statistics and bivariate analysis were performed to assess differences in characteristics in relation to the independent or dependent variables. Given that all variables assessed were categorical, chi-squared tests were used to determine differences of statistical significance. Unadjusted and adjusted survival analyses were performed using the Kaplan-Meier estimator and Cox proportional hazard regression models were used to determine the association between race, ethnicity, and the primary outcome-overall survival in months.

All statistical analyses were performed by SPSS version 22. This study was approved by the Kaiser Permanente Institutional Review Board for the Protection of Human Subjects, which has jurisdiction over the 21 hospitals described in this paper.

\section{Results}

\section{Patient characteristics}

As shown in Table 1, 2,470 (65.0\%) were Caucasian, 431 (11.3\%) were Hispanic, 342 (9.0\%) were African American, 538 (14.2\%) were Asian, and 18 (0.5\%) were Other. Statistically significant differences were found within age groups and the mean age of each race. The mean age of Caucasians was on average 2.5 years higher than the mean age of non-Caucasians ( $p<0.001)$. Caucasians also had the highest proportion of people greater than 70 years old $(41 \%)(p=0.006)$.

There was no significant difference in the location of the tumor between race groups ( $p=0.904)$, with approximately $25 \%$ of locations not specified across all races. Mean tumor size and number of lymph nodes examined were not reported in all patients and differences from available data were found to be not statistically significant.

There was no significant difference in the stage at time of diagnosis between race groups ( $p=0.953)$ and no significant difference in the different types of treatment received/ provided between race groups $(p=0.201)$. Upon utilizing multivariate analysis, all potential cofounders were not statistically significant except for the age group at time of diagnosis $(p=0.013)$ (Table 1 ).

\section{Overall survival}

Kaplan-Meier tests were used to analyze overall survival (OS) based on race and then age at diagnosis (Graph 1 and Graph 2). All race groups appear to follow a similar downward trend, with OS decreasing exponentially as time from diagnosis increased. The mean overall survival was 12.2 months for Caucasians, 15.7 months for Hispanics, 14.7 months for African Americans, 14.5 months for Asians, and 10.8 months for Other. While Caucasians and Other patients appear to have a lower mean OS, this difference is not statistically significant $(p=0.52)$ (Graph 1$)$.

However, Kaplan-Meier survival tests based on age group at time of diagnosis demonstrated a statistically significant difference in overall survival. All age groups appear to follow a similar downwards trend in overall survival from time of diagnosis, with OS decreasing exponentially as the number of months increased. The slope decreases more steeply with each increase in age group interval. The mean OS is 29.6 months for patients < 50 years old, 20.5 months for 50-59 years old, 16.0 months for 60-69 years old, 10.9 months 
Citation: Hsu DS, Le ST, Chang A, et al. (2021) Racial Parity in Pancreatic Cancer Treatment and Outcomes within an Integrated Healthcare System. J Pancreat Cancer Treat 3(1):14-21

Table 1: Patient variables.

\begin{tabular}{|c|c|c|c|c|c|c|}
\hline & Caucasian & Hispanic & African American & Asian & Other & \\
\hline$n$ & 2470 & 431 & 342 & 538 & 18 & 3799 \\
\hline$\%$ & 65 & 11.3 & 9 & 14.2 & 0.5 & \\
\hline \multicolumn{7}{|l|}{ Sex } \\
\hline Female & $48 \%$ & $49 \%$ & $54 \%$ & $51 \%$ & $17 \%$ & \\
\hline Male & $52 \%$ & $51 \%$ & $47 \%$ & $49 \%$ & $83 \%$ & $P=0.114$ \\
\hline \multicolumn{7}{|l|}{ Age Group } \\
\hline$<50$ & $3 \%$ & $7 \%$ & $5 \%$ & $6 \%$ & & \\
\hline $50-59$ & $12 \%$ & $15 \%$ & $14 \%$ & $15 \%$ & $22 \%$ & \\
\hline $60-69$ & $29 \%$ & $28 \%$ & $36 \%$ & $28 \%$ & $33 \%$ & \\
\hline 70-79 & $32 \%$ & $32 \%$ & $30 \%$ & $26 \%$ & $17 \%$ & \\
\hline$>80$ & $26 \%$ & $18 \%$ & $16 \%$ & $25 \%$ & $28 \%$ & $P<0.001$ \\
\hline Mean Age & 71.5 & 68.5 & 68.8 & 69.5 & 69.4 & $P<0.001$ \\
\hline$<70$ & $59 \%$ & $67 \%$ & $68 \%$ & $62 \%$ & $72 \%$ & \\
\hline 70 or Greater & $41 \%$ & $33 \%$ & $32 \%$ & $38 \%$ & $28 \%$ & $P=0.006$ \\
\hline \multicolumn{7}{|l|}{ Location } \\
\hline Body & $15 \%$ & $13 \%$ & $14 \%$ & $17 \%$ & $11 \%$ & \\
\hline Head & $48 \%$ & $49 \%$ & $47 \%$ & $47 \%$ & $44 \%$ & \\
\hline Tail & $15 \%$ & $16 \%$ & $14 \%$ & $13 \%$ & $11 \%$ & \\
\hline no & $22 \%$ & $22 \%$ & $25 \%$ & $24 \%$ & $33 \%$ & $P=0.904$ \\
\hline \multicolumn{7}{|l|}{ Tumor Size } \\
\hline Mean Tumor Size (mm) & 43.7 & 44.1 & 49.3 & 47 & 35.2 & $P=0.654$ \\
\hline$n$ & 1749 & 315 & 237 & 382 & 9 & \\
\hline \multicolumn{7}{|c|}{ Number of Nodes Examined } \\
\hline Mean Number of Nodes & 19.02 & 16.65 & 20.2 & 19.1 & 24.6 & $P=0.330$ \\
\hline$n$ & 436 & 89 & 64 & 96 & 5 & \\
\hline \multicolumn{7}{|l|}{ Stage } \\
\hline Localized & $10 \%$ & $9 \%$ & $8 \%$ & $9 \%$ & $6 \%$ & \\
\hline Regional Extension & $15 \%$ & $15 \%$ & $16 \%$ & $17 \%$ & $17 \%$ & \\
\hline Nodal & $25 \%$ & $24 \%$ & $24 \%$ & $26 \%$ & $22 \%$ & \\
\hline Distant & $53 \%$ & $53 \%$ & $52 \%$ & $52 \%$ & $44 \%$ & \\
\hline Unknown & $5 \%$ & $3 \%$ & $6 \%$ & $6 \%$ & $6 \%$ & $P=0.953$ \\
\hline \multicolumn{7}{|l|}{ Treatment } \\
\hline Surgery & $16.90 \%$ & $20.90 \%$ & $19.30 \%$ & $17.30 \%$ & $27.80 \%$ & \\
\hline Chemotherapy & $49.90 \%$ & $55.20 \%$ & $53.80 \%$ & $53.90 \%$ & $55.60 \%$ & \\
\hline Radiation & $8.10 \%$ & $10.20 \%$ & $8.80 \%$ & $8.20 \%$ & $11.10 \%$ & \\
\hline Surgery + Chemo & $10.40 \%$ & $11.10 \%$ & $9.90 \%$ & $12.10 \%$ & $16.70 \%$ & \\
\hline Surgery + Chemo + XRT & $3.10 \%$ & $4.40 \%$ & $4.70 \%$ & $2.80 \%$ & $11.10 \%$ & \\
\hline No Treatment & $45.50 \%$ & $38.70 \%$ & $40.40 \%$ & $42.20 \%$ & $44.40 \%$ & \\
\hline Neoadjuvant Chemo & $20.50 \%$ & $18.60 \%$ & $20.40 \%$ & $21.20 \%$ & $16.70 \%$ & $P=0.201$ \\
\hline
\end{tabular}


Citation: Hsu DS, Le ST, Chang A, et al. (2021) Racial Parity in Pancreatic Cancer Treatment and Outcomes within an Integrated Healthcare System. J Pancreat Cancer Treat 3(1):14-21

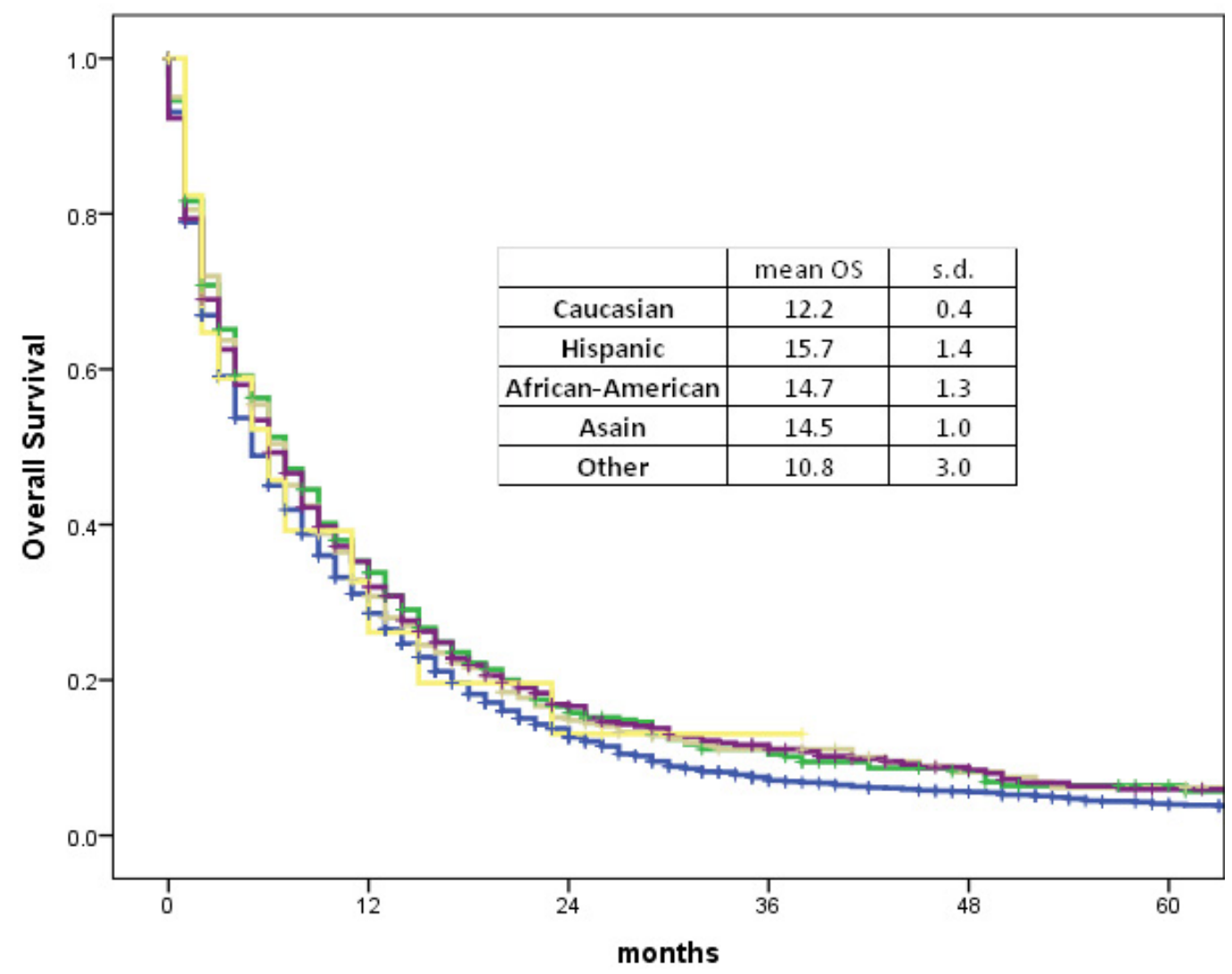

Graph 1: Race and survival.

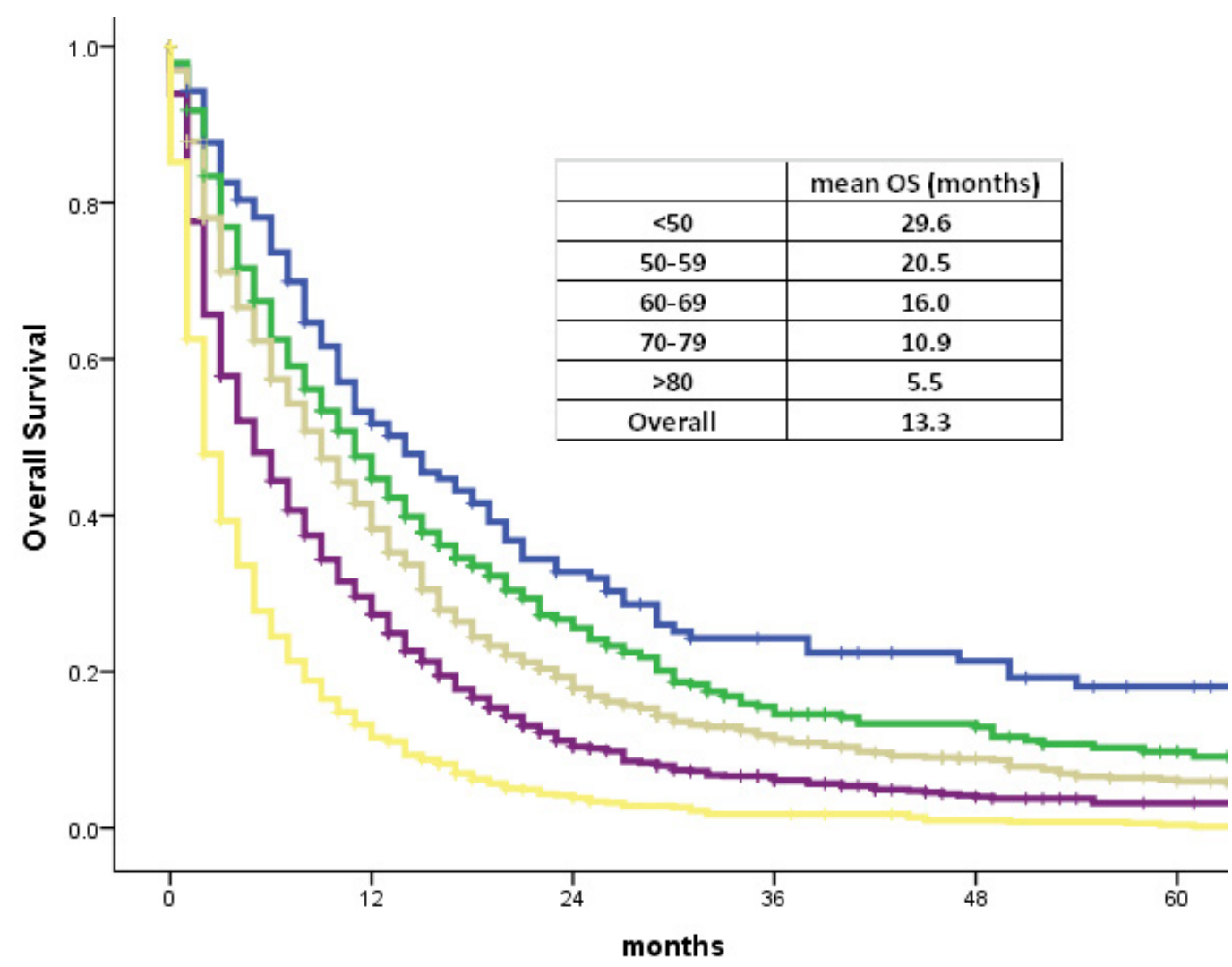

Graph 2: Age and survival. 
Citation: Hsu DS, Le ST, Chang A, et al. (2021) Racial Parity in Pancreatic Cancer Treatment and Outcomes within an Integrated Healthcare System. J Pancreat Cancer Treat 3(1):14-21

for $70-79$ years old, and 5.5 months for $>80$ years old. The difference in mean OS between age groups is statistically significant $(p<0.001)$ (Graph 2).

Patients were then analyzed based on stage of disease at time of diagnosis. The largest proportion of patients diagnosed with stages 1-3 from all race groups underwent no treatment ( $>25 \%$ for every race group). Excluding the Other race group due to the small sample size, the percentage of each race group receiving different treatment regimens were similar $(p=0.849)$. The difference in OS between the patients

Table 2: Therapy received at stage of disease.

\begin{tabular}{|c|c|c|c|c|c|c|}
\hline & Caucasian & Hispanic & African American & Asian & Other & \\
\hline \multicolumn{7}{|l|}{ Stage 1 to 3} \\
\hline $\mathrm{n}$ & 1052 & 188 & 142 & 230 & 9 & \\
\hline No Treatment & $30.70 \%$ & $25.50 \%$ & $27.50 \%$ & $26.50 \%$ & $33.30 \%$ & \\
\hline Surgery & $6.90 \%$ & $10.60 \%$ & $9.90 \%$ & $5.20 \%$ & & \\
\hline Chemo & $24.00 \%$ & $21.80 \%$ & $23.90 \%$ & $27.00 \%$ & $11.10 \%$ & \\
\hline Surgery + Chemo & $23.10 \%$ & $22.90 \%$ & $21.80 \%$ & $27.40 \%$ & $33.30 \%$ & \\
\hline Radiation & $1.50 \%$ & & $0.70 \%$ & $1.70 \%$ & & \\
\hline Radiation + Chemo & $6.70 \%$ & $9.00 \%$ & $4.90 \%$ & $5.70 \%$ & & \\
\hline Surgery + Chemo + XRT & $7.00 \%$ & $10.10 \%$ & $11.30 \%$ & $6.50 \%$ & $22.20 \%$ & $P=0.849$ \\
\hline \multicolumn{7}{|l|}{ Stage 4} \\
\hline$n$ & 1306 & 229 & 178 & 278 & 8 & \\
\hline No Treatment & $53.90 \%$ & $48.50 \%$ & $48.30 \%$ & $50.70 \%$ & $50.00 \%$ & \\
\hline OS Survival (months) & 6.1 & 7.9 & 6.6 & 6.9 & 4.5 & $P=0.115$ \\
\hline OS with Palliative Treatment & 10.7 & 12.3 & 10.4 & 11.6 & 7.3 & $P=0.503$ \\
\hline Treatment Age 75 or Greater & 7.9 & 8.8 & 7 & 8.5 & 5 & $P=0.922$ \\
\hline Treatment Age $<75$ & 11.3 & 13.1 & 11.4 & 12.5 & 8 & $P=0.548$ \\
\hline
\end{tabular}

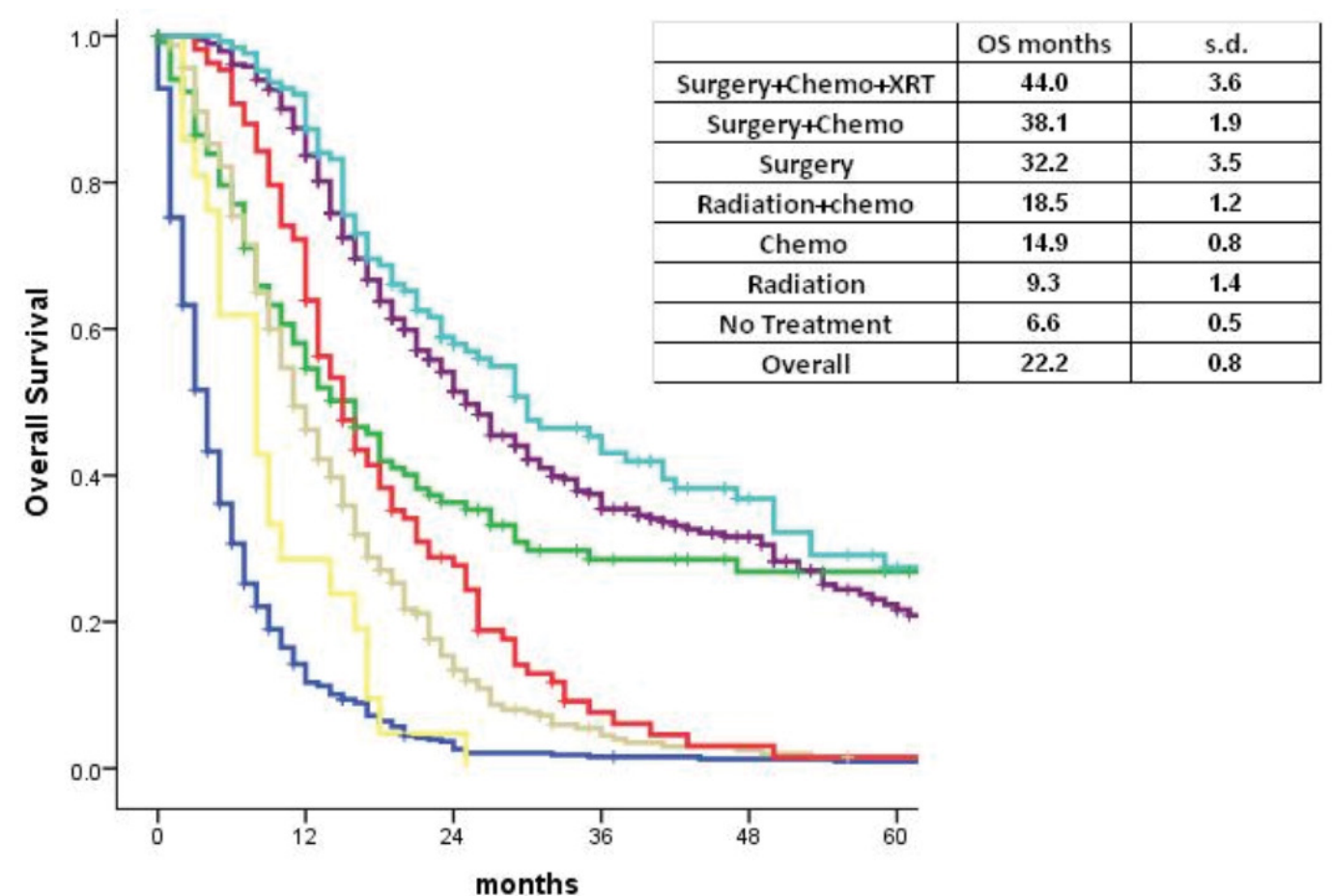

Graph 3: Therapy received at stage of disease. 
Citation: Hsu DS, Le ST, Chang A, et al. (2021) Racial Parity in Pancreatic Cancer Treatment and Outcomes within an Integrated Healthcare System. J Pancreat Cancer Treat 3(1):14-21

with stage 1-3 pancreatic cancer in the race groups was not statistically significant $(p=0.233)$.

Similarly, the largest proportion of stage 4 patients in each race group underwent no treatment ( $>48 \%$ for all race groups). Overall survival was significantly lower for stage 4 patients, averaging 6.4 months compared to the average 22.0 months for stage 1-3 patients across all race groups. The differences in OS for stage 4 pancreatic cancer patients of each race group were also not statistically significant ( $p=$ $0.115)$. Overall survival with palliative treatment was higher for all race groups, with no statistical difference ( $p=0.503$ ). Palliative treatment was provided more often for patients younger than 75 , and less likely for patients 75 or older, with no statistical difference between the different race groups ( $p$ $=0.548$ and $p=0.922$, respectively) (Table 2 ).

The overall survival was analyzed for patients with nonmetastatic disease undergoing different treatment therapies. The OS was highest, 44.0 months, for patients who received surgery, radiation, and chemotherapy, followed by those who received surgery and chemotherapy, 38.1 months. This difference in overall survival in these two treatment arms compared to other treatments was statistically significant ( $p$ $<0.001$ ) (Graph 3).

Overall survival was analyzed with Cox proportional hazards model and demonstrated no statistically significant difference among the different races. However, there was statistically significant difference for stage, treatment options, lymph node status, and age.

\section{Discussion}

Multiple studies have demonstrated worse survival and treatment inequality in African American patients diagnosed with pancreatic cancer [13-15]. This study demonstrates racial parity in pancreatic cancer treatment received and outcomes within a large integrated health system. Our study showed no difference in the percentage of African American patients who received surgery or other treatments compared to other race groups. Overall survival demonstrated no difference based on race. Differences in overall survival were seen along non-racial factors-such as age and treatments received.

The integrated delivery system unites a financing group with all providers from hospitals, clinics, physicians through home care and long-term care facilities to pharmacies $[16,17]$. KPNC is a consortium of three separate but interdependent entities: the Kaiser Foundation Health Plan and its regional operating organizations, Kaiser Foundation Hospitals, and the Permanente Medical Groups. Kaiser Foundation Health Plan and Hospitals are integrated with a legally separate physician group called Permanente Medical Groups. The health plan is the insurance part of the organization while the hospitals and medical group provide all clinical services $[18,19]$.

The public refers to these hospitals and physician groups as one organization, which is commonly referred to as "Kaiser". The health plan and hospitals operate under state and federal not-for-profit tax status, while the medical groups operate as for-profit partnerships or professional corporations in their respective regions. Patients have equal access to healthcare and ancillary services, such as imaging, referral to surgery, oncology, radiation, and chemotherapy regardless of type of insurance or facility at which they are seen.

In this study, statistically significant differences were seen when analyzing age at time of diagnosis in the different race groups. Our patient population was largely reflective of the demographics of the United States as a whole except for the Asian population, which was higher compared to national data, $14.2 \%$ vs. $5.9 \%$, and the African American population, which was lower than the national data, $9 \%$ vs. $13.4 \%$ [20]. However, our data population more closely reflected the population of California [21]. In our study, 32\% of Caucasians and Hispanics were diagnosed between the age of 70-79 whereas $36 \%$ of African Americans and $28 \%$ of Asians were diagnosed between the age of 60-69. The difference in age at time of diagnosis did not translate in differences in overall survival by race. Although this could imply that Caucasians were older at time of diagnosis and yet had no difference in survival compared to African American patients, both groups had $>80 \%$ diagnosed at $>60$ years old. The age distribution is consistent with other studies, demonstrating that $80 \%$ of pancreatic cancer is diagnosed in patients between the ages of $60-80[22,23]$.

In an integrated system like KPNC, the path to surgery is a streamlined process. If there is a positive radiologic finding, the imaging is sent to a hepatobiliary surgeon's EMR inbox. A surgeon is involved in the patient's care early regardless of whether or not this patient is a surgical candidate. Previous studies have looked at the role of patient navigation programs in improving patient-physician relations and times to diagnostic resolution [24]. Although KPNC does not have a staff member designated as a patient navigator or care coordinator for pancreatic cancer, the systems-based measures are able to link one step of the treatment process to another, much like a patient navigator or coordinator would.

Recent studies have shown that African American patients with resectable pancreatic cancer were less likely to be offered surgery and that they were more likely to refuse surgery [25]. Other studies have demonstrated similar findings that Black patients were less likely to receive surgery compared to White patients, but that this difference was not seen in patients who received neoadjuvant therapy $[26,27]$. In a paper by Nip, et al. (2018) looking at the SEER-Medicare database of pancreatic cancer patients, they found that older and African American and Hispanic patients had worse survival despite controlling for sociodemographic and clinical factors and older patients and African American patients were less likely to have surgery [15]. However, they also found that having any insurance coverage was the most important factor associated with undergoing surgery and that having any type of insurance, especially private insurance, was associated with better outcomes [25]. In our study, we did not look at differences in insurance type because every Kaiser member has equal access to physicians and ancillary services. However, patients have varying amounts of co-payment they are required to provide based on their insurance plan. Our study demonstrates that having insurance, regardless of plan type or coverage, and access to health care results in 
no difference in pancreatic cancer outcomes. In our study, there was no statistical difference between different race groups receiving different types of treatment (chemotherapy, radiation, surgery), thereby demonstrating that access to healthcare is an important equalizer in treatments provided to African American patients and minimizes the effects of bias in patient care. This ultimately resulted in no difference in survival in African American patients compared to other races.

Lam et al recently identified that overall mortality from nine types of cancer-surgery specific mortality and postoperative complications decreased over 10 years for both Black and White patients but that the mortality gap between Black and White patients did not change. These results imply that overall care, treatment, and technique for surgical oncology has improved but that there is still a difference in care between races and this has not been addressed by these improvements. They were interested in seeing a shift in care from lower to higher-quality hospitals for Black patients, but this was not identified [28]. Our study is unable to address the systemic, nationwide disparities in outcomes based on lower and higher quality hospitals, but the equalization of treatments provided, and survival outcomes here suggest that insurance status, treatment at a higher-volume hospital, or both can help bridge the difference between Black and White patients and perhaps will spur policy changes to accomplish higher rates of health insurance.

The main limitation of this study is that our population is largely comprised of people with health insurance (private, Medicare, Medical). Some studies have suggested that African American race is a placeholder for lower socioeconomic (SES) status, thereby suggesting that pancreatic cancer outcomes are linked to SES instead of race. 2019 Census data showed that $9.6 \%$ and $16.7 \%$ of African Americans and Hispanics, respectively, compared to $5.2 \%$ of non-Hispanic Whites, did not have health insurance. Of those without health insurance, $16 \%$ were below the poverty line whereas of those who were at or above $400 \%$ of the poverty line, only $3 \%$ were without health insurance [29]. Although patients included in this study may have different co-payments, the cohort analyzed in this study is limited by its inability to include the segment of the population without insurance. Thus, the differences in SES among different race groups, particularly the most impoverished, were unable to be adequately captured by the scope of this study.

Second, our primary outcome and patient characteristics analyzed were limited to survival and age, race, disease stage, and therapy received, respectively. There may be other factors, such as readmission and complication rates, that were not investigated and yet are important indicators of outcome as well. Studies have identified higher complication rates in postoperative Black patients [28]. Patient characteristics such as comorbidities score and surgery performed were not analyzed here but have been included in other studies in order to assess patient outcomes [30,31].

A third limitation is the retrospective nature of the study and a patient cohort that is not population-based, thereby limiting the generalizability of results. During this time, the regionalization of pancreatic cancer care was implemented at KPNC and increasing utilization of neoadjuvant chemotherapy occurred. These are all potential confounding factors not addressed within this study.

\section{Conclusion}

This study demonstrates that in an integrated health system, where there is equal access to health care, there is no difference in survival outcomes based on race for pancreatic adenocarcinoma patients. This is different from previous publications. Age of diagnosis and treatments received significantly affected overall survival; younger patients and those who received the most aggressive treatment regimen (surgery, chemotherapy, and radiation) had longer overall survival. In addition, the improved overall survival seen with multimodal treatment is an important area of further research to standardize neoadjuvant chemoradiation for pancreatic cancer [32,33].

\section{Acknowledgements}

None.

\section{Funding}

None.

\section{References}

1. ACS C (2014) Cancer facts and figures. American cancer society.

2. Zhang C, Zhang C, Wang Q, et al. (2020) Differences in stage of cancer at diagnosis, treatment, and survival by race and ethnicity among leading cancer types. JAMA Netw Open 3: e202950.

3. Jinjuvadia R, Jinjuvadia K, Liangpunsakul S (2013) Racial disparities in gastrointestinal cancers-related mortality in the U.S. population. Dig Dis Sci 58: 236-243.

4. Shah A, Chao KS, Ostbye T, et al. (2013) Trends in racial disparities in pancreatic cancer surgery. J Gastrointest Surg 17: 1897-1906.

5. Murphy MM, Simons JP, Hill JS, et al. (2009) Pancreatic resection. Cancer 115: 3979-3990.

6. Lucas FL, Stukel TA, Morris AM, et al. (2006) Race and surgical mortality in the United States. Ann Surg. 243: 281-286.

7. Siegel RL, Miller KD, Jemal A (2015) Cancer statistics, 2015. CA Cancer J Clin 65: 5-29.

8. Lim JE, Chien MW, Earle CC (2003) Prognostic factors following curative resection for pancreatic adenocarcinoma: A populationbased, linked database analysis of 396 patients. Ann Surg 237: 74-85.

9. Fesinmeyer $\mathrm{MD}$, Austin $\mathrm{MA}, \mathrm{Li} \mathrm{Cl}$, et al.(2005) Differences in Survival by Histologic type of pancreatic cancer. Cancer Epidemiol Biomarkers Prev 14: 1766-1773.

10. Revels SSL, Banerjee M, Yin H, (2013) Racial disparities in surgical resection and survival among elderly patients with poor prognosis cancer. J Am Coll Surg 216: 312-319.

11. Arnold LD, Patel AV, Yan Y, (2009) Are racial disparities in pancreatic cancer explained by smoking and overweight/ obesity? Cancer Epidemiol Biomarkers Prev 18: 2397-2405.

12. Patel AV, Rodriguez C, Bernstein L, et al. (2005) Obesity, recreational physical activity, and risk of pancreatic cancer in 
a large U.S. Cohort. Cancer Epidemiol Biomarkers Prev14: 459466.

13. Riall TS, Townsend CM, Jr., Kuo YF, et al.( 2010) Dissecting racial disparities in the treatment of patients with locoregional pancreatic cancer: A 2-step process. Cancer 116: 930-939.

14. Singal V, Singal AK, Kuo YF (2012) Racial disparities in treatment for pancreatic cancer and impact on survival: A populationbased analysis. J Cancer Res Clin Oncol 138: 715-722.

15. Nipp R, Tramontano AC, Kong CY, et al. (2018) Disparities in cancer outcomes across age, sex, and race/ethnicity among patients with pancreatic cancer. Cancer Med 7: 525-535.

16. Coddington DC, Moore KD, Fischer EA (1994) Integrated health care: Reorganizing the physician, hospital, and health plan relationship. Med Group Manage J 40: 76-78.

17. Gröne O, Garcia-Barbero M (2001) Integrated care: A position paper of the WHO european office for integrated health care services. Int J Integr Care 1:e21.

18. Strandberg-Larsen M, Schiøtz ML, Frølich A (2007) Kaiser permanente revisited-. Paper presented at: Meeting the health care needs of tourists.

19. Towill DR (2006) Viewing Kaiser Permanente via the logistician lens. Int J Health Care Qual Assur Inc Leadersh Health Serv 19: 296-315.

20. Facts ACQ (2015) US Census Bureau.

21. Sathyamurthy A, Chela H, Romana B, et al. (2016) Pancreatic cancer survival outcomes at a tertiary medical center based on sex and geographic location. South Med J 109: 718-720.

22. Wang H, Liu J, Xia G, et al. (2020) Survival of pancreatic cancer patients is negatively correlated with age at diagnosis: A population-based retrospective study. Scientific reports 10 : 7048.
23. Ilic M, Ilic I (2016) Epidemiology of pancreatic cancer. World J Gastroenterol 22: 9694 -9705.

24. Rodday AM, Parsons SK, Snyder F, et al. (2015) Impact of patient navigation in eliminating economic disparities in cancer care. Cancer. 121: 4025-4034.

25. Moaven O, Richman JS, Reddy S, et al. (2019) Healthcare disparities in outcomes of patients with resectable pancreatic cancer. Am J Surg 217: 725-731.

26. Molina G, Clancy TE, Tsai TC, et al. (2021) Racial disparity in pancreatoduodenectomy for borderline resectable pancreatic adenocarcinoma. Ann Surg Oncol 28: 1088-1096.

27. Heller DR, Nicolson NG, Ahuja N, et al. (2020) Association of treatment inequity and ancestry with pancreatic ductal adenocarcinoma survival. JAMA Surg 155: e195047.

28. Lam MB, Raphael K, Mehtsun WT, et al. (2020) Changes in racial disparities in mortality after cancer surgery in the US, 20072016. JAMA Netw Open 3: e2027415.

29. Berchick ER, Hood E, Barnett JC (2018) Health insurance coverage in the United States: 2017. Current population reports Washington DC: US Government Printing Office.

30. Wegner RE, Verma V, Hasan S, et al. (2019) Incidence and risk factors for post-operative mortality, hospitalization, and readmission rates following pancreatic cancer resection. J Gastrointest Oncol 10:1080-1093.

31. Sadot E, Brennan MF, Lee SY, et al. (2014) Readmission after pancreatic resection: Causes and causality pattern. Ann Surg Oncol 2: 4342-4350.

32. Springfeld C, Jäger D, Büchler MW, et al.(2019) Chemotherapy for pancreatic cancer. Presse Med 48: e159-e174.

33. Landau E, Kalnicki $S$ (2018) The evolving role of radiation in pancreatic cancer. Surg Clin North Am 98: 113-125.

DOI: $10.36959 / 829 / 400$

Copyright: (c) 2021 Hsu DS, et al. This is an open-access article distributed under the terms of the Creative Commons Attribution License, which permits unrestricted use, distribution, and reproduction in any medium, provided the original author and source are credited. 